\title{
POLÍTICA FISCAL, EXPECTATIVAS Y TRANSICIÓN DINÁMICA EN EL MODELO SIMPLE DE CRECIMIENTO ENDÓGENO
}

\author{
Alejandro Rodríguez Arana*
}

(Recibido: noviembre 2013/Aceptado: abril 2014)

\section{Resumen}

En el modelo neoclásico simple de crecimiento endógeno este artículo muestra que las políticas fiscales que podrían considerarse expansivas propician un nivel de acervo de capital de largo plazo más elevado cuando son anunciadas que cuando no lo son. Sin embargo, el efecto de estas políticas sobre la tasa de crecimiento de largo plazo es el mismo independientemente de si son o no anunciadas. En particular, los cambios en las tasas de impuestos al consumo son neutrales cuando no son anunciados, pero tienen efectos de corto plazo sobre el crecimiento y permanentes sobre el acervo de capital cuando son anunciados.

Palabras clave: crecimiento endógeno, política fiscal, expectativas racionales, ecuación de Euler.

Clasificación JEL: E21 E22 E62.

\begin{abstract}
In the simplest neoclassical endogenous growth model, this paper shows that fiscal policies that could be considered as expansive produce a higher

* Profesor-investigador de la Universidad Iberoamericana, plantel Ciudad de México, DF, Prolongación, Paseo de la Reforma núm. 880, col. Lomas de Santa Fe, México DF, CP. 01219, tel. 59-50-42-68. >alejandro.rodriguez@uia.mx>.
\end{abstract}


level of the capital stock when they are announced. The effects of these policies on the long rate of growth are the same whether or not the policies are announced, nonetheless. In particular, changes in the consumption's tax rates are neutral when they are not announced, but they have short run effects on the rate of growth, and a permanent effect on the capital stock, when they are announced.

Keywords: endogenous growth, fiscal policy, rational expectations Euler equation.

JEL Classification: E21 E22 E62.

\section{Introducción}

La discusión sobre los efectos de la política fiscal en el crecimiento económico tiene muchos años. Harberger (1962) (1964) sugiere neutralidad de los impuestos sobre el crecimiento, posición defendida aún en tiempos más recientes por diversos economistas (Mendoza et al. 1997). En modelo exógeno, como el de Solow (1956), los efectos de los impuestos sobre el crecimiento son, en el mejor de los casos, temporales pues en el largo plazo el ingreso per cápita se expande a la tasa exógena de crecimiento de la productividad factorial.

En 1991 Rebelo (1991) propone un modelo endógeno en el cual diversas variables fiscales afectan la tasa de crecimiento del producto de largo plazo. La tasa del impuesto sobre la renta propicia una caída al reducir el rendimiento efectivo del capital. Sin embargo, la tasa del impuesto al consumo carece de efectos sobre el mismo.

En los últimos veinte años, diversos modelos endógenos respaldan en general la idea de que la tasa del impuesto sobre la renta reduce el crecimiento y que la tasa del impuesto al consumo tiene efectos nulos sobre aquella variable, véase: Kim (1992), Jones, Manuelli y Rossi (1993) Stokey y Rebelo (1993). Sin embargo, otros modelos encuentran resultados diferentes; Kaneko y Matsuzaky (2009) señalan que en presencia de una demanda de dinero la tasa del impuesto al consumo sí puede afectar el crecimiento de largo plazo; Day y Chengyu (2011) muestran condiciones por las cuales una misma variable fiscal puede tener efectos diversos sobre el crecimiento dependiendo de otras condiciones de la economía; Haruyama e Itaya (2006) indican que en algunos casos, cuando hay fuertes imperfecciones, 
la presencia de múltiples tasas impositivas en el impuesto sobre la renta, o en los impuestos al consumo, puede generar mayor crecimiento de largo plazo.

A nivel empírico la relación entre la política fiscal y el crecimiento de largo plazo no presenta aún un patrón definitivo. Easterley y Rebelo (1992) discuten la dificultad de aislar el efecto de las tasas impositivas sobre el crecimiento. Algunos estudios empíricos favorables a la relación de las variables fiscales con el crecimiento son los de Alper y Cakici (2009), Afonso y Furceri (2010), Bhattariai (2010) y Segura-Ubiergo et al. (2010). Trabajos menos favorables a la relación de largo plazo entre las variables fiscales y el crecimiento son los de Kaliva y Tunaru (2007) y Colombier (2009).

Un aspecto poco analizado en la relación de la política fiscal y el crecimiento es el papel que juegan las expectativas. Una política que aumenta la tasa de algún impuesto puede tener efectos diversos sobre la acumulación de capital dependiendo si es o no anunciada. Las expectativas de un cambio de política en el futuro probablemente afecten el crecimiento de corto plazo, o incluso el de largo plazo, pero en todo caso es casi seguro que tendrán efectos permanentes sobre el acervo de capital.

El objetivo principal de este trabajo es analizar los efectos de diversas variables de política fiscal sobre el crecimiento económico tanto cuando los cambios en dichas variables son anunciados como cuando no lo son. Para ello, la segunda sección de este trabajo describe una versión ligeramente modificada del modelo original de Rebelo (1991), poniendo especial atención en sus propiedades dinámicas. La tercera sección analiza los efectos de cambios no anunciados en diversas variables fiscales. En seguida se analizan los efectos de cambios anunciados en esas mismas variables.

Los resultados del artículo muestran que una política fiscal que podría considerarse expansiva (reducción de tasas impositivas y aumento del consumo público como proporción del producto) tiene mayores efectos positivos permanentes sobre el acervo de capital cuando es anunciada que cuando no lo es. Sin embargo, el efecto de largo plazo de las políticas anunciadas sobre las tasas de crecimiento del producto y el capital es el mismo que el de las políticas no anunciadas. Esto implica que las políticas anunciadas tienen efectos sobre las tasas de crecimiento del producto y el capital por el simple hecho de anunciarse. 


\section{El modelo}

Partimos del modelo de Rebelo (1991), donde el producto es proporcional a un capital agregado, el cual incluye no sólo capital físico sino también capital humano. Este modelo es conocido como $A K$.

$$
Y_{t}=A K_{t}
$$

$A$ es la productividad marginal del capital. $K$ es el capital agregado y $Y$ el producto.

La idea de que el producto es proporcional al capital no es nueva ni tampoco es de Rebelo, como él mismo lo reconoce (Rebelo, 1991: 501). En su descripción sobre los datos estilizados del crecimiento en el mundo, Kaldor (1961) señala que para diversos países desarrollados el cociente entre el producto y el capital a lo largo del tiempo es bastante constante. El modelo de Harrod y Domar (Harrod (1948), Domar (1946)) supone una relación lineal entre el producto y el capital. También lo supone así Pasinetti (1960). Para algunos autores (D'Alessandro y Salvadori, 2008) el modelo $A K$ es una reedición del modelo clásico de David Ricardo.

Los empresarios maximizan beneficios:

$$
B_{t}=\left(A K_{p t}-r_{t} K_{p t}-\delta K_{p t}\right)(1-\tau)
$$

Donde $K_{p t}$ es el capital en poder del sector privado, $r$ es la tasa de interés o el costo del capital y $\delta$ es la tasa de depreciación del capital, la cual se supone constante. $\tau$ es la tasa del impuesto sobre la renta. En teoría, los beneficios de los empresarios están sujetos al pago del impuesto sobre la renta.

El capital total de la economía está dividido entre el sector privado y el sector público:

$$
K_{t}=K_{p t}+K_{p u t}
$$

Donde $K_{p u t}$ es el capital de la economía en manos del sector público.

La maximización de beneficios en (2) da por resultado:

$$
r_{t}=A-\delta
$$


La tasa de interés es igual al beneficio marginal neto del capital $(A-\delta)$. Los beneficios señalados en (2) son cero y el producto privado se agota en el pago al capital y la depreciación del capital del sector privado.

\subsection{Maximización de la utilidad de los consumidores}

Por el lado del consumo, los consumidores maximizan la función de utilidad intertemporal:

$$
\int_{0}^{\infty} U\left(C_{t}\right) e^{-\theta t} d t
$$

Donde $\theta$ es la tasa subjetiva de descuento.

La maximización está sujeta a que en cada momento en el tiempo los ingresos netos de los individuos menos su gasto en consumo generan acumulación de capital y activos financieros.

$$
\begin{gathered}
r_{t}\left(K_{p t}+b_{t}\right)(1-\tau)+S_{t}-\left(1+\tau_{c}\right) C_{t}=\left(1+\tau_{a}\right)\left(\frac{d K_{p t}}{d t}+\frac{d b_{t}}{d t}\right) \\
K_{p t}+b_{t}=V_{t}
\end{gathered}
$$

Donde $S$ son subsidios netos del tipo lump sum del gobierno al sector privado; $b$ son bonos públicos en poder del sector privado; $k_{p}+b=V$ es la riqueza total privada; $\tau_{c}$ es la tasa del impuesto al consumo; $\tau_{a}$ es la tasa del impuesto al ahorro que, aunque tal vez no exista en muchos países, es teóricamente posible. Los bonos públicos $b$ rinden la misma tasa de interés que el capital agregado. La tasa de impuesto a la acumulación de capital es la misma que la de acumulación de bonos, de otra manera nadie acumularía alguno de los dos activos.

El problema dinámico de maximización se lleva a cabo a través de establecer el hamiltoniano:

$$
\operatorname{Max} H=U\left(C_{t}\right)+\lambda_{t}\left(r_{t} \frac{(1-\tau)}{\left(1+\tau_{a}\right)} V_{t}+\frac{S_{t}}{\left(1+\tau_{a}\right)}-\frac{\left(1+\tau_{c}\right)}{\left(1+\tau_{a}\right)} C_{t}\right)
$$

$C$, el consumo privado, es la variable de control; $V$ es la variable de estado y $\lambda$, el multiplicador de Lagrange, es la variable de coestado. 
Las condiciones de primer orden de este problema son (Blanchard y Fischer, 1989: 38-40).

$$
\begin{gathered}
U_{c t}-\lambda_{t} \frac{\left(1+\tau_{c}\right)}{\left(1+\tau_{a}\right)}=0 \\
\left.\frac{d \lambda_{t}}{d t}-\theta \lambda_{t}=-r_{t} \frac{(1-\tau)}{\left(1+\tau_{a}\right.}\right) \lambda_{t} \\
\lim _{t \rightarrow \infty} \lambda_{t} V_{t} e^{-\theta t}=0
\end{gathered}
$$

Donde $U_{c}$ es la utilidad marginal de consumo $d U / d C$.

(9) es la condición usual de equimarginalidad en el consumo. (10) es la condición dinámica del modelo de optimización. (11) es la condición de transversalidad, la cual señala que en el largo plazo la riqueza no puede crecer por arriba de la tasa subjetiva de descuento $\theta$.

De la primera condición:

$$
\lambda_{t}=\frac{\left(1+\tau_{a}\right)}{\left(1+\tau_{c}\right)} U_{c t}
$$

Sustituyendo esta ecuación en la segunda condición y rearreglando términos, se obtiene:

$$
\frac{d C_{t}}{d t}=\left(\theta-r_{t} \frac{(1-\tau)}{\left(1+\tau_{a}\right)}\right) \frac{U_{c t}}{U_{c c t}}
$$

(13) es la condición dinámica del consumo para cualquier función de utilidad. $U_{c c}$ es la primera derivada de la utilidad marginal respecto al consumo $U_{c} / U_{c c}$ es el coeficiente de aversión al riesgo.

Utilizaremos la función de utilidad isoelástica, donde la utilidad instantánea se define como:

$$
U_{t}=\frac{C_{t}^{1-\frac{1}{\rho}}}{1-\frac{1}{\rho}}
$$

Donde $\rho$ es la elasticidad de sustitución en el consumo intertemporal. Obteniendo las derivadas correspondientes a (14) y sustituyendo en (13) se obtiene: 


$$
\frac{d C_{t}}{d t} \frac{1}{C_{t}}=g_{c t}=\rho\left(\frac{r_{t}(1-\tau)}{\left(1+\tau_{a}\right)}-\theta\right)
$$

La ecuación (15) muestra la trayectoria óptima del consumo para la función de utilidad isoelástica. $g_{c}$ es la tasa de crecimiento del consumo. El consumo crece más conforme más elevada sea la elasticidad de sustitución $(\rho)$ y menor sea la tasa subjetiva de descuento $\theta$. La tasa del impuesto sobre la renta y la tasa de impuestos al ahorro reducen el crecimiento del consumo.

Sustituyendo la ecuación (4) en (15) se obtiene:

$$
g_{c}=\rho\left(\frac{(A-\delta)(1-\tau)}{\left(1+\tau_{a}\right)}-\theta\right)
$$

Para tasas de impuestos a la renta y al ahorro con un valor constante se encuentra que el crecimiento del consumo es también constante.

\subsection{La restricción presupuestal del sector público y el equilibrio general}

El sector público tiene los siguientes gastos e ingresos:

$$
\text { gastos }=S_{t}+G_{t}+r_{t} b_{t}+\frac{d K}{d t}
$$

Donde $G$ es el consumo público y $d k_{p u} / d t$ es la acumulación de capital del sector público.

Por otra parte, los ingresos del sector público son:

$$
\text { ingresos }=r_{t} K_{\text {put }}+\tau r_{t}\left(K_{p t}+b_{t}\right)+\tau_{c} C_{t}+\tau_{a}\left(\frac{d K_{p t}}{d t}+\frac{d b}{d t}\right)
$$

El primer término corresponde a los ingresos públicos por producción del propio sector público; el segundo término muestra los ingresos por impuesto sobre la renta; el tercer término son los ingresos por impuestos al consumo; el cuarto término son los ingresos públicos por impuestos al ahorro privado.

La resta de (17) y (18) es el déficit público, el cual se financia con emisión de bonos $d b / d t$.

Combinando el déficit público con la restricción presupuestal del sector privado en (6) se obtiene:

$$
A K_{t}=C_{t}+G_{t}+\frac{d K}{d t}+\delta K_{t}
$$


Que es la ecuación del PIB a costo de factores. El PIB $A K$ es igual al consumo total $C+G$ más la inversión bruta total $d K / d t+\delta K$.

Dividiendo (19) entre el capital total y re arreglando términos:

$$
g_{k t}=(A-\delta)-\left(C_{k t}+G_{k t}\right)
$$

Donde $C_{k}$ es la razón de consumo privado a capital total; $G_{k}$ es la razón de consumo público a capital total; $g_{k}$ es la tasa de crecimiento del capital total, la cual es igual a la tasa de crecimiento del producto pues éste es proporcional al capital. La ecuación (20) indica que el crecimiento del capital de esta economía es igual al producto interno neto por unidad de capital $(A-\delta)$ menos el consumo total de la economía también por unidad de capital $\left(C_{k}+G_{k}\right)$.

La gráfica 1 muestra las ecuaciones (16) y (20).

\section{Gráfica 1}

El crecimiento y la razón consumo privado-capital del equilibrio

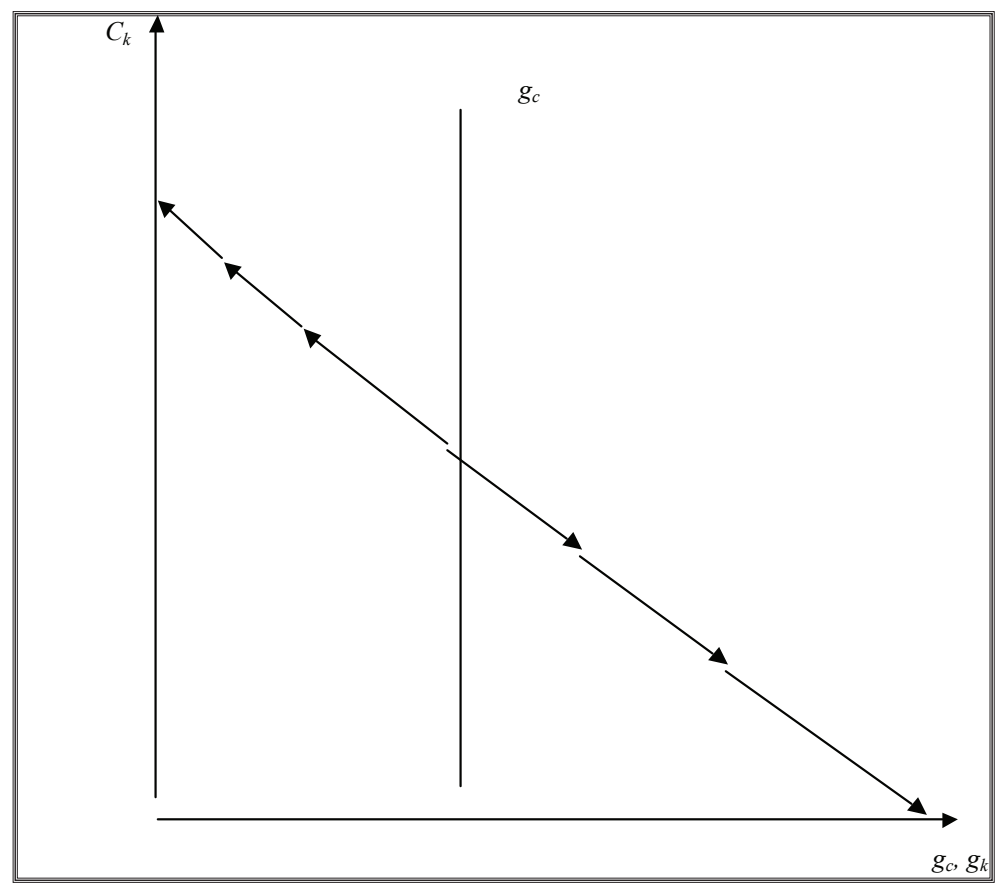

Fuente: elaboración propia. 
La ecuación (16) se representa por la línea vertical. El crecimiento del consumo privado es constante. La línea con pendiente negativa representa la ecuación (20). Es una recta con $45^{\circ}$ de pendiente.

La intersección entre las dos funciones es un punto estacionario donde hay crecimiento balanceado. En ese punto el crecimiento de capital y del producto son iguales al crecimiento del consumo, el cual es constante y determinado por los parámetros de la ecuación (16).

La economía debe estar siempre sobre la recta con pendiente negativa. El modelo presenta una dinámica inestable desde el punto de vista tradicional, como por ejemplo en el lenguaje de Chiang (1984). A la izquierda de la recta vertical el crecimiento del consumo es mayor que el crecimiento del capital, lo que implica que la razón de consumo privado a capital $\left(C_{k}\right)$ está creciendo y el crecimiento del capital se hace todavía menor que el del consumo. A la derecha de la línea vertical el crecimiento del capital es mayor que el crecimiento del consumo, por lo cual la razón $C_{k}$ está cayendo y eso propicia un crecimiento del capital todavía mayor que el crecimiento del consumo, lo que refuerza la inestabilidad.

La característica de inestabilidad tradicional en este modelo es deseable. La razón de esto es que tanto el cociente de consumo privado a capital $\left(C_{k}\right)$ como el crecimiento del capital $\left(g_{k}\right)$ son variables no predeterminadas. Una variable predeterminada no puede moverse en forma discontinua. En cambio, una variable no predeterminada sí puede hacerlo, es decir puede saltar en un momento dado en el tiempo (Buiter, 1982).

Si el modelo aquí presentado fuera estable en el sentido tradicional, entonces tendría infinitas soluciones al equilibrio pues las variables endógenas $g_{k}$ y $C_{k}$ podrían iniciar en cualquier punto - puesto que no están predeterminadas- y llegar de ahí al equilibrio. En cambio, si el modelo ha de estar en equilibrio, y las variables no están predeterminadas, la inestabilidad implica que sólo hay una solución hacia el equilibrio. En este caso, si un parámetro cambia en forma no anticipada, el modelo pasa del equilibrio inicial de crecimiento balanceado al nuevo punto de equilibrio también balanceado. Es decir, para cambios no anticipados en los parámetros la aparente inestabilidad del modelo hace que éste opere como un modelo estático.

La literatura económica ofrece diversos ejemplos de modelos con la característica de inestabilidad global en el sentido tradicional. El más antiguo, tal vez, es el modelo de demanda de dinero de Sargent y Wallace (1973), otro ejemplo es el de Drazen (1985) sobre el problema de aritmética incómoda 
(unpleasant arithmetic). Blanchard y Fischer (1989, capítulo 5) muestran varios ejemplos, uno concreto también sobre la demanda de dinero (Blanchard y Fischer, 1989: 241) más recientemente, el nuevo modelo IS-LM también presenta la característica de inestabilidad global al trabajar con dos variables no predeterminadas: el producto y la inflación, como por ejemplo Woodford (2001).

La aparente inestabilidad de estos modelos es una condición necesaria para poder trabajar con expectativas. Cambios anticipados en los parámetros tienen efectos aunque todavía no hayan ocurrido. En términos matemáticos esto es posible modelarlo justamente por el rasgo de inestabilidad de los modelos. Una vez que se anuncia un cambio, el modelo deja de estar en el equilibrio estático y toma una ruta inestable por un periodo de tiempo hasta que el cambio esperado se concreta. Esto no sería posible hacerlo en un modelo globalmente estable.

En el caso que nos ocupa, Rebelo (1991) obtiene la solución de equilibrio de largo plazo, donde en el modelo lineal simple que estamos analizando los crecimientos del capital y del producto son iguales al crecimiento constante del consumo. Sin embargo, ni él ni otros autores ${ }^{1}$ analizan las propiedades dinámicas del modelo. Los efectos dinámicos son importantes pues las expectativas pueden modificar en forma permanente las trayectorias del capital, el producto y el consumo.

Las trayectorias inestables pueden ser factibles en el corto plazo pero no en el largo plazo. La gráfica 1 muestra que la trayectoria que se aleja del equilibrio hacia abajo puede descartarse como solución de largo plazo porque implicaría tener un consumo privado igual a cero o negativo, lo cual no es económicamente posible. La trayectoria que se aleja del equilibrio hacia arriba implica que tarde o temprano se tendrían tasas de crecimiento del capital tan negativas que la economía terminaría colapsándose, lo cual tampoco es factible. Por esta razón la única solución viable de largo plazo es el equilibrio balanceado.

\section{Política fiscal en el modelo de crecimiento endógeno}

Esta sección analiza los efectos de las variables fiscales sobre el crecimiento económico y, en general, sobre las trayectorias del capital, el producto y el consumo.

\footnotetext{
${ }^{1}$ Por ejemplo, Jones y Manuelli (1990), Easterley y Rebelo (1992) y Kaneko y Matsuzaki (2009).
} 
3.1 Los efectos de cambios permanentes no anunciados en las variables fiscales

Para hacer el análisis más expedito reproducimos las ecuaciones (16) y (20).

$$
\begin{aligned}
g_{c} & =\rho\left(\frac{(A-\delta)(1-\tau)}{\left(1+\tau_{a}\right)}-\theta\right) \\
g_{k t} & =(A-\delta)-\left(C_{k t}+G_{k t}\right)
\end{aligned}
$$

En donde la ecuación (21) muestra el crecimiento del consumo, la línea vertical en la gráfica 1, y la ecuación (22) muestra el crecimiento del capital o la línea con pendiente negativa en la gráfica 1 . Las variables fiscales que intervienen en estas ecuaciones son la tasa del impuesto sobre la renta $\tau$, la tasa del impuesto al ahorro $\tau_{a}$ y el consumo público como proporción del capital total $G_{k}$.

Los efectos de cambios permanentes no anunciados en estas variables pueden analizarse combinando las ecuaciones (21) y (22) con la gráfica 1.

La tasa del impuesto sobre la renta tiene un efecto negativo sobre el crecimiento del consumo y, en equilibrio, sobre el crecimiento del capital y del producto. Esto puede observarse en la ecuación (21) y en la condición de largo plazo de equilibrio balanceado. Un aumento de esta tasa reduce la tasa de interés neta que reciben los consumidores $\mathrm{y}$, por lo tanto, estimula el consumo, generando menor ahorro y menor crecimiento. Este resultado ya ha sido descrito por Rebelo (1991).

Un efecto muy similar ocurre con la tasa del impuesto al ahorro. Esta tasa es equivalente a un impuesto a la tasa de interés y, por lo tanto, estimula el consumo privado y disminuye el ahorro generando menor crecimiento económico en el equilibrio.

Los efectos gráficos de un incremento en la tasa del impuesto sobre la renta, o de la tasa de impuesto al ahorro, son un desplazamiento de la función $g_{c}$ hacia la izquierda. El crecimiento del capital cae y la razón consumo capital aumenta.

La otra variable fiscal es el consumo público como proporción del capital. Si esta variable aumenta, la línea recta con pendiente negativa, que representa a la ecuación (22), se desplaza hacia abajo. El crecimiento del consumo queda constante en todo momento, al igual que el crecimiento del capital. En cambio la razón de consumo privado a capital cae en exactamente lo mismo que aumentó la razón de consumo público a capital. Por lo cual hay solamente un efecto desplazamiento (crowding out) de 100\% que deja el consumo total inalterado. 
Otras variables fiscales no aparecen directamente en la ecuación. La tasa del impuesto al consumo es neutral al crecimiento y la acumulación de capital, tal como Rebelo (1991) también lo muestra. En cuanto a la inversión, es claro que si todos los parámetros están constantes $g_{k}$ también es constante. Un aumento de la inversión pública debe entonces provocar un efecto desplazamiento (crowding out) de uno a uno sobre la inversión privada. Los subsidios tipo lump sum tampoco están en las ecuaciones y no tienen efectos sobre el crecimiento y la acumulación de capital.

Una forma de neutralizar el impacto del impuesto sobre la renta consiste en que la tasa del impuesto al ahorro se vuelva negativa y con el mismo valor absoluto de la tasa del impuesto sobre la renta. Esto puede observarse tomando la ecuación (21) y haciendo que:

$$
\frac{1-\tau}{1+\tau_{a}}=1
$$

De aquí:

$$
\tau_{a}=-\tau
$$

Si éste es el caso, entonces el crecimiento de largo plazo será:

$$
g_{c}=\rho(A-\delta-\theta)
$$

Y ninguna variable fiscal tendrá efectos sobre el crecimiento y la acumulación de capital.

Si se establece una tasa de subsidio al ahorro en bonos y a la acumulación de capital igual a la tasa del impuesto sobre la renta, esto es equivalente a deducir dicho ahorro en bonos y tal acumulación de capital del pago del impuesto sobre la renta. Esto puede observarse cuando en la restricción presupuestal del sector privado (ecuación (6)) se impone la condición (24). En tal caso dicha ecuación (6) se convierte en:

$$
\left(r_{t}\left(K_{p t}+b_{t}\right)-\left(\frac{d K_{p t}}{d t}+\frac{d b_{t}}{d t}\right)\right)(1-\tau)+S_{t}-\left(1+\tau_{c}\right) C_{t}=0
$$


En el mundo actual hay diversos esquemas que emulan esta solución. En México, por ejemplo, el llamado impuesto especial a tasa única (IETU) puede deducir las inversiones en capital físico del ingreso. Sin embargo, la solución teórica de este trabajo muestra que habría que deducir no sólo la acumulación de capital físico, sino también la de capital humano (educación) y la acumulación en general de activos financieros. Si no se hiciera así, se estaría castigando tanto la acumulación de capital humano como la de activos financieros. En el modelo aquí descrito nadie compraría un bono público adicional si la acumulación de capital pudiera deducirse de los ingresos pero no el ahorro en bonos. Eso podría llevar a la quiebra del gobierno. En un contexto más realista poder deducir la acumulación de bonos públicos, pero no otros activos financieros, generaría salidas masivas de recursos del mercado financiero privado hacia el sector público, lo que pondría en riesgo al sistema financiero mismo.

\subsection{Efectos de cambios anunciados en las variables fiscales}

Cambios anunciados y creíbles en algunas variables fiscales pueden alterar el crecimiento económico desde antes de que el cambio se lleve a cabo. Esto propiciará una alteración permanente en la trayectoria de los acervos de capital cuando ésta se compara con el caso en el cual el cambio no fue anunciado.

Lo anterior sucede porque los consumidores suavizan el consumo lo más posible. Ante un cambio no anunciado, por ejemplo, una reducción de la tasa del impuesto sobre la renta (gráfica 2), el equilibrio entre la razón de consumo-capital y el crecimiento del capital y del consumo pasa instantáneamente de $A$ a $D$. El consumo se ajusta bruscamente porque nadie esperaba que fuera a ocurrir un cambio en la tasa impositiva. 
Gráfica 2

Análisis de una reducción anunciada y no anunciada en la tasa del impuesto sobre la renta

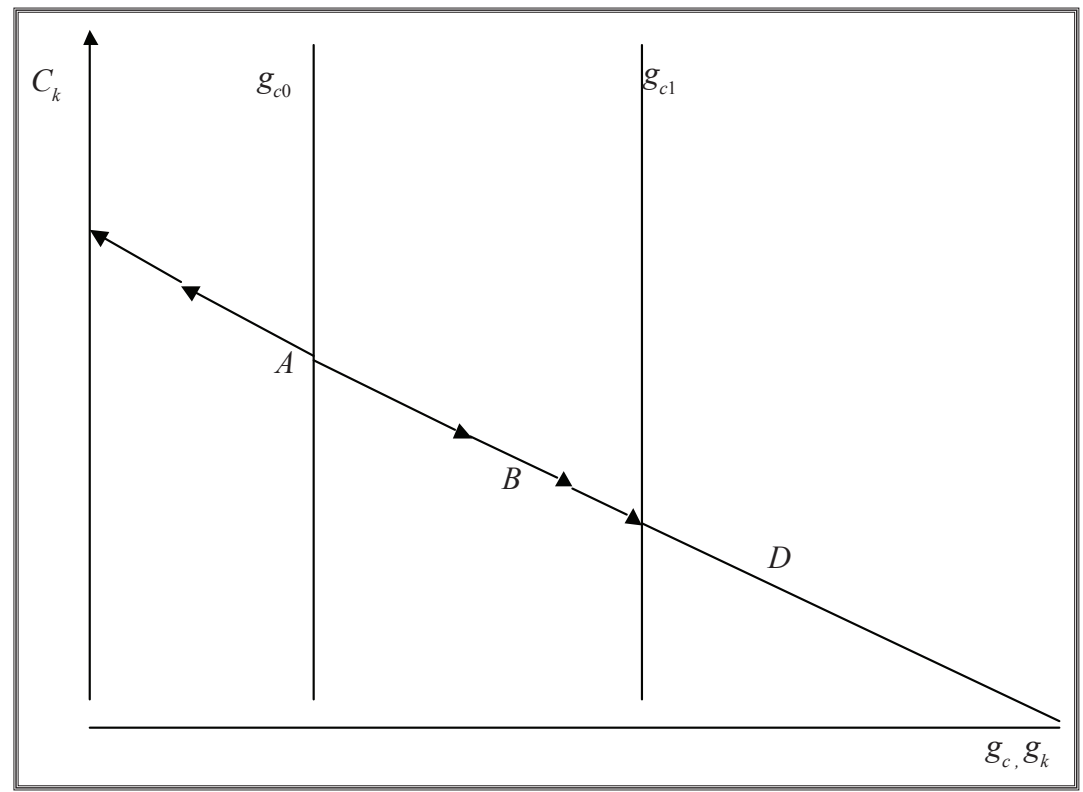

Fuente: elaboración propia.

En contraste, un cambio anunciado con anterioridad va a implicar un ajuste más pausado del consumo. Si en el momento $t_{0}$ se anuncia que en el momento futuro $t_{1}$ habrá una reducción en la tasa del impuesto sobre la renta, la gente sabe que en el futuro el consumo se tendrá que ajustar a la baja en algún momento. El objetivo de un consumo con una trayectoria más suave hace que el ajuste brusco se lleve a cabo desde el momento $t_{0}$. En ese momento el consumo cae de modo que la razón consumo capital pasa de $A$ a $B$. En $B$ la reducción de la tasa del impuesto sobre la renta no ha ocurrido, por lo cual la dinámica es inestable. La razón $C_{k}$ sigue cayendo. En el momento $t_{1}$ la razón $C_{k}$ está en el punto $D$. En ese instante se lleva a cabo la reducción de la tasa del impuesto sobre la renta, el crecimiento del consumo aumenta de $g_{c 0}$ a $g_{c 1}$ y la economía queda atrapada en el nuevo equilibrio $D$. La solución de este tipo de problema es muy similar a la descrita por Sargent y Wallace (1973) en su artículo clásico de expectativas racionales.

Al final, la razón $C_{k}$ y el crecimiento del capital $g_{k}$ terminan en el mismo nivel tanto con el cambio no anunciado como con el anunciado. Sin embargo, 
los niveles de consumo y de capital totales van a terminar siendo más elevados en el caso del cambio anunciado que en el no anunciado. La razón es muy simple: en el caso del cambio anunciado, entre el periodo $t_{0}$ y $t_{1}$ la economía creció a una tasa más elevada que en el caso del cambio no anunciado.

La gráfica 3 muestra las trayectorias del logaritmo del capital tanto para el caso anunciado como para el caso no anunciado. En el caso no anunciado, el crecimiento del capital es el inicial hasta el periodo $t_{1}$. A partir de ese momento el crecimiento aumenta de una sola vez. Cuando la política se anuncia en el periodo $t_{0}$ para comenzar en el periodo $t_{1}$, el capital comienza a crecer a una tasa más elevada desde $t_{0}$, por lo cual la acumulación de capital es mayor que en el caso no anunciado. A partir de $t_{1}$ el crecimiento del capital es el mismo en ambos casos, pero en el caso anunciado el capital acumulado es mayor y lo será siempre con respecto al caso no anunciado.

Gráfica 3

Trayectorias del capital con reducciones anunciada y no anunciada de la tasa del impuesto sobre la renta

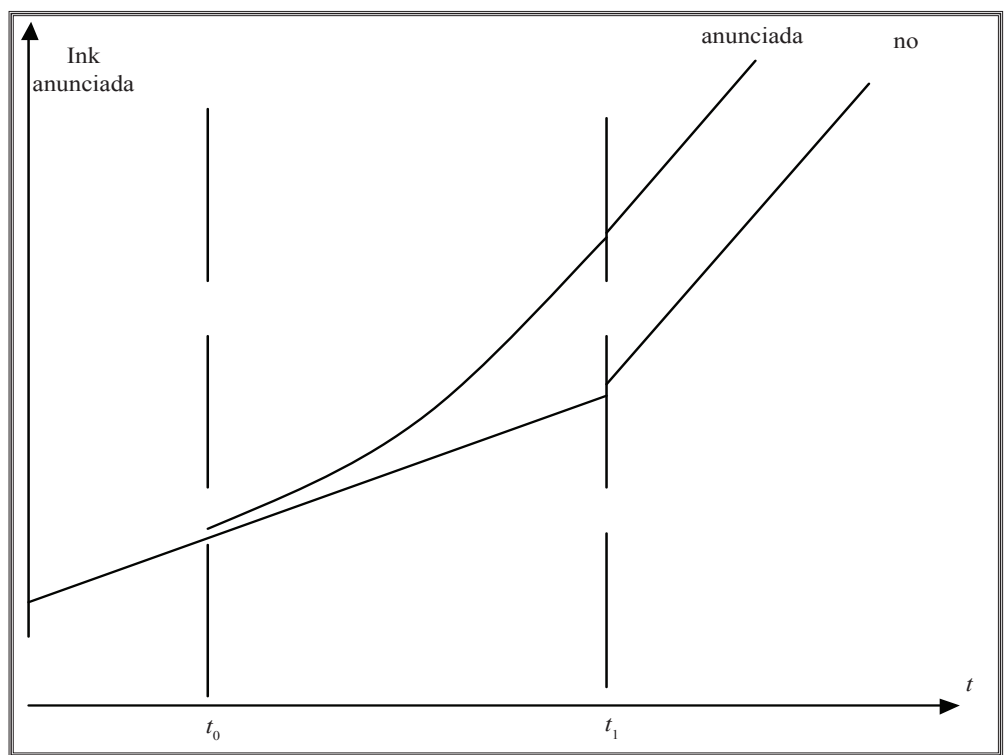

Fuente: elaboración propia.

Por su parte, la gráfica 4 muestra las trayectorias del logaritmo del consumo en los dos casos analizados. En el caso no anunciado el consumo lleva una trayectoria suave, con un crecimiento constante, entre un periodo inicial 
dado $\left(t_{0}\right)$ y el momento $t_{1}$. En ese momento, la tasa del impuesto sobre la renta cae en forma sorpresiva y el consumo se desploma del punto $D$ al $F$ para luego crecer a la nueva tasa de crecimiento mayor.

En el caso anunciado, el consumo cae en el momento $t_{0}$ (de $A$ a $B$ en la gráfica 4 ) en el cual se anuncia la reducción de la tasa en $t_{1}$. La tasa de crecimiento del consumo entre $t_{0}$ y $t_{1}$ es la misma que antes de $t_{0}$. En $t_{1}$ el consumo está en E- un punto superior a F- Esto se debe a que en ese momento la razón de consumo a capital en ambos casos es la misma pero el capital del caso anunciado es mayor. A partir de ahí el crecimiento del consumo es el nuevo y el mismo que en el caso anunciado, pero dado que la base del consumo en $t_{1}$ es mayor en el caso anunciado que en el caso no anunciado, el consumo del caso anunciado siempre será mayor que el del caso no anunciado.

El anuncio de una reducción en la tasa del impuesto sobre la renta tiene un impacto positivo temporal en el crecimiento del capital, el cual propicia una trayectoria para el capital y el consumo de largo plazo mayores que en el caso donde no se anuncia la política.

\section{Gráfica 4}

Trayectorias del consumo con reducciones anunciada y no anunciada de la tasa del impuesto sobre la renta

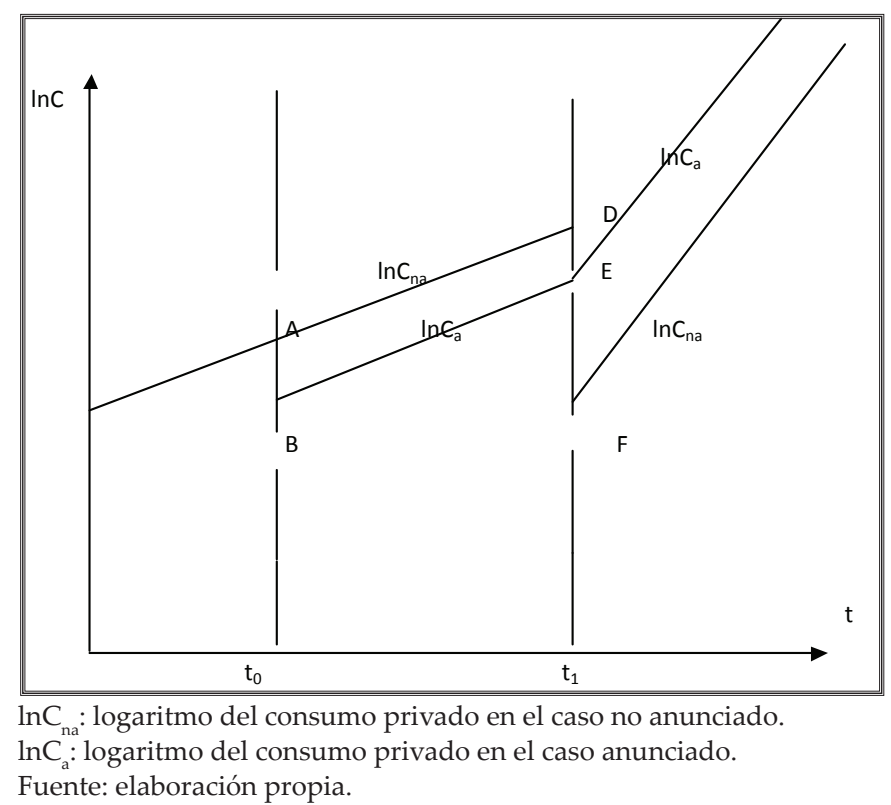


El anuncio de cambios en otras variables fiscales también puede tener efectos permanentes sobre el capital y el consumo de largo plazo. Estos efectos son diferentes al caso donde no existe dicho anuncio. Así, por ejemplo, si se anuncia que en el futuro habrá un incremento del consumo público como proporción del producto o del capital, eso traerá una reducción del consumo privado presente y un aumento temporal del crecimiento económico. La razón de esto es que el público espera una caída del consumo privado como proporción del capital de largo plazo, por lo que la caída se daría ahora, antes de que se lleve a cabo el aumento del consumo público como proporción del capital. En este caso habría una trayectoria más elevada para el capital que en el caso donde no se anuncia la política, que es una situación donde no se altera en ningún momento la acumulación de capital (véase apéndice).

El hecho de que un anuncio de política pueda lograr por sí sólo un efecto deseado, puede dar lugar a un problema de inconsistencia temporal (Buiter y Miller, 1982). Si anunciado una caída de la tasa del impuesto sobre la renta se propicia un mayor crecimiento, puede no haber un incentivo para el gobierno de llevar a cabo la política anunciada una vez que la gente espera que dicha política tenga lugar. Aunque lo óptimo en el momento del anuncio sea llevar a cabo la política, es posible que tal práctica no sea óptima cuando llega el momento de hacerlo porque el anuncio de hacerlo ya logró incrementar el acervo de capital y en forma permanente. El equilibrio de inconsistencia temporal sería uno donde el anuncio no es creíble desde el principio pues la gente entiende que, de ser creíble, los incentivos del gobierno para llevar a cabo la política ya no serían los mismos en el momento en que debería llevarse a cabo.

Buiter y Miller, (1982) discuten un caso similar en el tema de la inflación. Cuando el banco central promete llevar a cabo una política monetaria restrictiva en el futuro y la gente lo cree, la inflación comienza a ceder desde hoy pues la expectativa de inflación es menor y la demanda de dinero aumenta (mismo modelo que el clásico de Sargent y Wallace, 1973). Entonces es posible que el banco central pierda los incentivos de llevar a cabo la política monetaria restrictiva porque con el simple anuncio de hacerlo ya logró lo que deseaba, bajar la inflación. El problema entonces es que el anuncio no es creíble desde el principio y la inflación no cede.

Sin embargo, diversos análisis (Barro y Gordon, 1983) muestran que es posible lograr la credibilidad de las políticas a través de compromisos en 
los cuales el responsable de la política se impone un castigo en caso de no cumplir con lo prometido. Si quien promete la reducción de la tasa impositiva, por ejemplo un candidato al gobierno que tomará posesión en el futuro, firma un compromiso en el cual su castigo sería renunciar a la presidencia en caso de no cumplir lo prometido, la política se vuelve creíble y los resultados de las expectativas resultan permanentes.

3.3. Sobre la no neutralidad de un cambio esperado en la tasa del impuesto al consumo

De acuerdo a Rebelo (1991), los impuestos al consumo son neutrales al crecimiento económico. El modelo de Rebelo no tiene explícitamente dinero como activo. Kaneko y Matsuzaki (2009) muestran que cuando el consumo y el dinero tienen algún grado de sustitución, los impuestos al consumo no son neutrales en términos del crecimiento económico.

En el marco del modelo original de Rebelo (1991) es posible mostrar que cambios esperados en la tasa del impuesto al consumo tienen un efecto temporal sobre la tasa de crecimiento del capital y permanente sobre el acervo de capital. La intuición de este resultado es relativamente fácil de comprender. Si se espera que la tasa del impuesto al consumo suba en el futuro, va a haber una sustitución intertemporal de consumo futuro por consumo presente, pues los bienes de consumo en el presente son relativamente más baratos que en el futuro. El incremento del consumo presente reduce la acumulación de capital del presente.

En términos del modelo descrito en este trabajo tomemos la ecuación (9) cuando $\tau_{a}=0 .^{2}$ En este caso:

$$
U_{c t}=\lambda_{t}\left(1+\tau_{c t}\right)
$$

Asimismo, la ecuación (10) señala que la tasa de crecimiento del multiplicador de Lagrange $\lambda$ es constante, por lo cual:

$$
\frac{d \lambda}{d t} \frac{1}{\lambda}=\left(\theta-r_{t}(1-\tau)\right)=(\theta-(A-\delta)(1-\tau))
$$

\footnotetext{
${ }^{2}$ Este supuesto lo hacemos para simplificar el álgebra pero el resultado no se afecta siempre y cuando $\tau_{a}$ sea constante.
} 
De aquí que entre el momento $t_{0}$, en el cual se comienza a esperar que la tasa del impuesto al consumo cambie, y $t_{1}$, en el cual efectivamente la tasa cambia, sucede que:

$$
\frac{U_{c t 1}}{\left(1+\tau_{c 1}\right)}=\frac{U_{c t 0}}{\left(1+\tau_{c 0}\right)} e^{(\theta-((A-\delta)(1-\tau)))\left(t_{1}-t_{0}\right)}
$$

Donde $\tau_{c 0}$ es la tasa del impuesto al consumo que está en vigor entre $t_{0}$ y $t_{1}$ y $\tau_{c 1}$ es la tasa al impuesto al consumo que está en vigor a partir de $t_{1}$. Suponemos $\tau_{c 1}>\tau_{c 0}$.

Utilizando la función de utilidad isoelástica (ecuación (14)) se llega a:

$$
C_{t 0}^{\frac{1}{\rho}}\left(1+\tau_{c 0}\right)=C_{t 1}^{\frac{1}{\rho}}\left(1+\tau_{c 1}\right) e^{(\theta-(A-\delta)(1-\tau))\left(t_{1}-t_{0}\right)}
$$

Donde $C_{t 0}$ es el consumo exactamente en el momento $t_{0}$ y $C_{t 1}$ es el consumo en el momento $t_{1}$.

Tomando logaritmos de ambos lados y re arreglando términos: ${ }^{3}$

$$
\ln C_{t 1}-\ln C_{t 0}=-\rho\left(\tau_{c 1}-\tau_{c 0}\right)+\rho((A-\delta)(1-\tau)-\theta)\left(t_{1}-t_{0}\right)
$$

La diferencia entre los niveles de consumo entre el momento $t_{0}$ y $t_{1}$ está influenciada por la diferencia entre las tasas de impuestos al consumo.

Para tener una mejor comprensión de la ecuación (31), supongamos que el crecimiento de largo plazo de la economía es cero. Esto implica que el último término del lado derecho de tal ecuación es también cero, pues justamente ese término representa al crecimiento balanceado de largo plazo. Si ése fuera el caso, y la tasa impositiva nunca cambiara, el consumo permanecería constante. En cambio si $\tau_{c 1}>\tau_{c 0}$ el consumo en el periodo $t_{0}$ sería superior al del periodo $t_{1}$. La diferencia entre las tasas impositivas explica la sustitución intertemporal en el consumo, $C_{t 0}$ aumenta.

$\mathrm{El}$ incremento en el consumo en el periodo actual, debido a la expectativa de un aumento futuro de impuestos al consumo, reduciría la acumulación de capital de cero a un nivel negativo. Por lo cual, en la transición el acervo de capital estaría cayendo. Al llegar al momento $t_{1}$, el consumo tendría que caer, pero no podría regresar al nivel inicial anterior a $t_{0}$ porque el acervo de

\footnotetext{
${ }^{3}$ Estamos suponiendo que $\tau_{c}$ es una magnitud pequeña, de modo que $\ln \left(1+\tau_{c}\right)=\tau_{c}$.
} 
capital es ahora menor, lo que implica un consumo final por abajo del que tenía lugar antes de $t_{0}$. En el momento $t_{1}$ el crecimiento pasaría de negativo a cero.

Cuando se considera una tasa de crecimiento de largo plazo positiva y constante, la solución es muy similar. En lugar de cero hay una tendencia de consumo representada por el crecimiento constante de largo plazo. La expectativa de una tasa de impuesto al consumo más elevada en el futuro, propicia un incremento del consumo presente por arriba de la tendencia. Después del incremento súbito del consumo en $t_{0}$, esta variable sigue creciendo a la tasa de largo plazo. No obstante, el mencionado incremento de una vez por todas reduce la tasa de crecimiento del capital, por lo cual la acumulación de capital cae por debajo de su tendencia. En el momento $t_{1}$ el consumo cae súbitamente, y tiene que caer por debajo de la tendencia pues el capital acumulado es menor que el que hubiera tenido lugar en ausencia de cualquier cambio esperado. A partir de su nuevo nivel, el consumo sigue creciendo a la tasa de largo plazo. El lector puede observar que el consumo nunca creció a una tasa distinta que la de equilibrio, sólo tuvo dos cambios súbitos, uno hacia arriba en $t_{0}$ y otro hacia abajo en $t_{1}$.

La trayectoria del capital se ve afectada negativamente. Antes del momento $t_{0}$ el capital crece a la tasa de largo plazo $\rho((A-\delta)(1-\tau)-\theta)$. En $t_{0}$ el consumo aumenta, por lo cual la razón de consumo capital también aumenta. Esto en la gráfica 1 puede verse como pasar del equilibrio a un punto a la izquierda de la recta vertical $g_{c}$ sobre la línea con pendiente negativa. La tasa de crecimiento del capital $g_{k}$ cae, por lo cual el cociente de consumo a capital sigue subiendo y la tasa $g_{k}$ sigue bajando. En $t_{1}$ hay un nuevo cambio súbito del consumo por el cual la razón $C_{k}$ regresa al equilibrio estacionario de la gráfica. A partir de ahí el capital vuelve a crecer a la tasa de largo plazo.

La reducción temporal del crecimiento del capital provoca un efecto permanente negativo en el acervo de capital al compararlo con el caso en el cual el aumento en la tasa del impuesto al consumo no es esperado.

La solución que hemos dado a este problema es muy similar a la que propone Calvo (1986) para un tema diferente. En el artículo mencionado, Calvo muestra que cuando hay una inflación futura esperada más elevada que la actual, hay una sustitución intertemporal en la cual el consumo presente aumenta y el futuro disminuye. Esto se debe a la 
existencia de una restricción de efectivo (cash in advance), en la cual el consumo y el dinero resultan altamente complementarios. En épocas donde la inflación es relativamente baja la gente demanda más dinero y más consumo, por eso si la inflación esperada futura es más elevada que la actual, el consumo presente está por arriba de su tendencia.

\section{Conclusiones}

Los resultados de este trabajo muestran que las políticas fiscales anunciadas y no anunciadas tienen efectos permanentes sobre el acervo de capital que son diferentes entre sí. En general una política fiscal que pudiera considerarse expansiva (reducción de tasas impositivas, aumento del consumo público como proporción del producto) tiene efectos positivos sobre al acervo de capital -si es que los tiene- más elevados que una política fiscal expansiva no anunciada. Lo mismo pasa, pero en sentido contrario, con las políticas fiscales restrictivas. Las que son anunciadas deterioran más el acervo de capital que las no anunciadas.

Una posible implicación de este resultado es que habría incentivos para los gobiernos de anunciar políticas fiscales expansivas, porque generan un incremento adicional sobre el acervo de capital, y no anunciar u ocultar políticas fiscales restrictivas, porque de hacerlo habría un deterioro adicional sobre al acervo de capital. Esto podría generar un problema de extracción de señales (signal extraction), en el cual el silencio del gobierno podría dar lugar a la percepción de que existe una probabilidad positiva de ajuste fiscal futuro, en cuyo caso aunque el gobierno no tuviera la intención de llevar a cabo esa política, podría haber efectos nocivos para el acervo de capital.

En los trabajos futuros en este tema deberían plantearse estos problemas probablemente en el marco de la teoría de juegos. Tal vez podría encontrarse un esquema óptimo de anuncios de política fiscal en que el acervo de capital sufra lo menos posible.

El artículo aquí presentado parte de un esquema simplificado y aun así su solución tiene algunas complejidades. La introducción de externalidades muy posiblemente no cambiaría los resultados principales. Un análisis con otros elementos, como la oferta de trabajo o la acumulación de dinero en efectivo, podría generar resultados diferentes y sería de interés llevarlo a cabo. 
Un aspecto que muestra el presente trabajo es que, aun cuando sean falsas, las expectativas de un cambio futuro afectan la acumulación de capital presente. Tal vez por esta razón algunos trabajos empíricos, como los de Easterley y Rebelo (1992), encuentren tantas dificultades en el análisis de la relación de diversas variables fiscales y el crecimiento económico. Si se pudiera controlar el cambio de la variable fiscal con el hecho de haber sido o no anunciada, probablemente se podría llegar a conclusiones más exactas. En esto también hay un reto para el análisis econométrico.

Finalmente, durante años se ha señalado que las tasas de impuestos al consumo son neutrales al crecimiento y la acumulación de capital. Sin embargo, esto sucede cuando los movimientos de dichas tasas son inesperados. Los movimientos esperados de las tasas de impuestos al consumo pueden tener efectos muy grandes en el crecimiento de cortomediano plazo y en el acervo de capital de largo plazo.

Apéndice: aumentos anticipado y no anticipado del consumo público como proporción del capital

Partimos de las ecuaciones (20) y (21) del texto, que son las que se grafican en el plano donde en el eje vertical está el consumo privado como proporción del capital $\left(C_{k}\right)$ y en el eje horizontal están la tasa de crecimiento del capital $g_{k}$ y la tasa de crecimiento del consumo $g_{c}$.

$$
\begin{aligned}
& g_{c}=\rho\left(\frac{(A-\delta)(1-\tau)}{\left(1+\tau_{a}\right)}-\theta\right) \\
& g_{k t}=(A-\delta)-\left(C_{k t}+G_{k t}\right)
\end{aligned}
$$


El equilibrio inicial está en el punto $A$ de la gráfica A.1. Si el aumento en el consumo público como proporción del capital $\left(G_{k}\right)$ es no anunciado, el equilibrio pasa de $A$ a $E$ de una sola vez y no se afecta en ningún momento ni el crecimiento del capital ni el acervo de capital.

\section{Gráfica A.1}

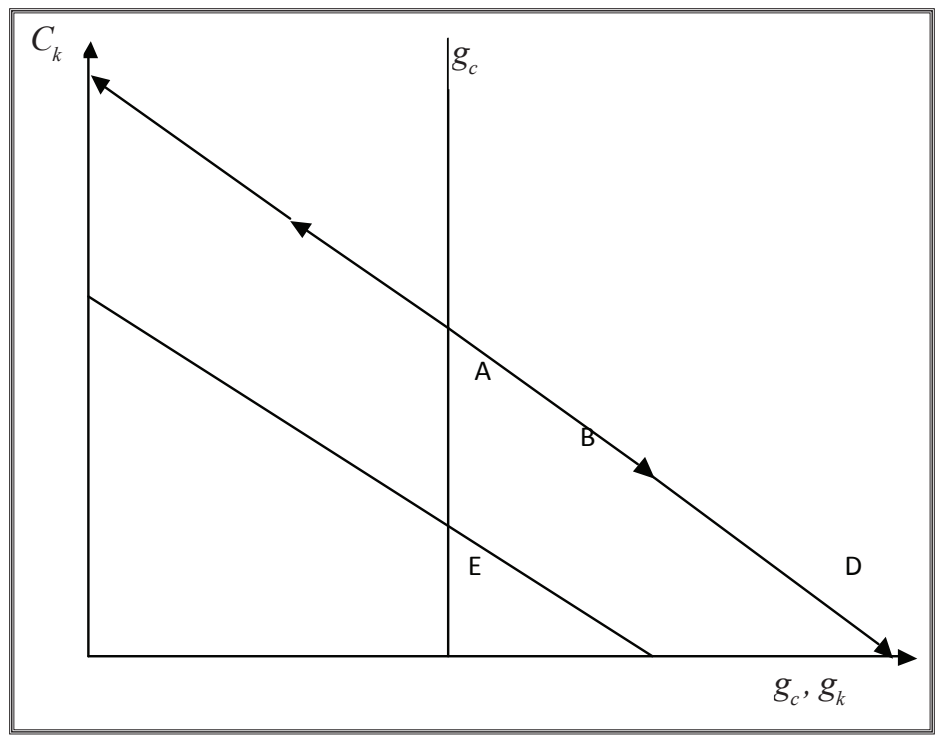

Fuente: elaboración propia.

Cuando el aumento del consumo público es anunciado, el consumo privado se suaviza lo más posible. El salto inicial del consumo es de $A$ a $B$, pues el cambio no ha tenido lugar. En $B$ la ruta inestable lleva a la economía a mayor crecimiento del capital y menor razón de consumo privado a capital. Cuando el sistema está en $D$ - un punto paralelo a $E$ - ocurre el cambio y el crecimiento del capital salta del punto $D$ al punto $E$ con un consumo privado como proporción del capital que en ese momento queda predeterminado.

El incremento temporal del crecimiento del capital propicia un aumento permanente del acervo de capital, cuya trayectoria es diferente y mayor que la del caso del cambio no anunciado. 


\section{Referencias}

Afonso, A y D. Furceri (2010). “Government Size, Composition, Volatility and Economic Growth". European Journal of Political Economy, vol. 26, 517-532.

Alper, C. y S. Cakici (2009). "Financial Liberalization, Fiscal Prudence and Growth: Panel Evidence From 1980-2003”. Open Economies Review, vol. 20, 509-524.

Barro, R. y D. Gordon (1983). "A Positive Theory of Inflation in a Natural Rate Model”. Journal of Political Economy, 91, pp. 589-610.

Bhattarai, K. (2010). “Taxes, Public Spending and Economic Growth in OECD countries". Problems and Perspectives in Management, vol. 8, 14-30.

Blanchard, O. y S. Fischer (1989). Lectures on Macroeconomics. MIT Press. Cambridge, Massachusetts.

Buiter, W. (1982). "Predetermined and Non Predetermined Variables in Rational Expectations Models". NBER Technical paper series 21. National Bureau of Economic Research.

Buiter, W. y M. Miller (1985). “Costs and Benefits of Anti-Inflationary Policy: Questions and Issues", en V. Angry y J. Neville (comps.), Inflation and Unemployment: Theory, Experience and Policy Making. Londres, G. Allen and Unwin.

Calvo, G (1986). “Temporary Stabilization: Predetermined Exchange Rates”. Journal of Political Economy, vol. 94, 1319-1329.

Chiang, A. (1984). Fundamental methods of mathematical economics. Tokyo, McGraw-Hill. International Student Edition.

Colombier, C. (2009). "Growth Effects of Fiscal Policies: An Application of Robust Modified M Estimator". Applied Economics, vol. 41, 899-912.

D’Alessandro, S. y N. Salvadori (2008). “Pasinetti versus Rebelo: Two Different Models or Just One?". Journal of Economic Behavior and Organization, vol. 65, 547-574.

Day, R (2011). "Economic Growth and the Effects of Fiscal Policy". Metroeconomica, vol. 62, 218-234.

Domar, E. (1946). "Capital Expansion, Rate of growth, and Employment". Econometrica, vol. 14, 137-147.

Drazen, A. (1985). "Tight Money and Inflation: Further results". Journal of Monetary Economics, vol. 15, 113-120.

Easterley, W. y S. Rebelo (1992). “Marginal Income Tax Rates and Economic Growth in Developing Countries". The World Bank, Policy Research Working Paper Series 1050. Washington D. C., World Bank. 
Harberger, A. (1962). "The Incidence of the Corporation Income Tax". Journal of Political Economy, vol. 70, 215-240. (1964). "Taxation, Resource Allocation and Welfare", en J.F. Due (comp.), The Role of Direct and Indirect Taxes in the Federal Revenue System. Princeton, Princeton University Press.

Harrod, R. (1948). Towards a dynamic economics. Londres, McMillan.

Haruyama, T. y J. Itaya (2006). “Do Distortionary Taxes Always Harm Growth?" Journal of Economics, vol. 87, 99-126.

Jones, L. y R. Manuelli (1990). “A Convex Model of Equilibrium Growth: Theory and Policy Implications". Journal of Political Economy, vol. 98, 1008-1038.

Jones, L. R. Manuelli y P. Rossi (1993). “Optimal Taxation in Models of Endogenous Growth". Journal of Political Economy, vol. 101, 485-517.

Kaliva, A. y R. Tunaru (2007). “Economic Growth and Indirect Financial Taxes: Empirical Evidence from Greece, Spain and Portugal". Frontiers in Finance and Economics, vol. 4, 47-74.

Kaldor, N. (1961). "Capital Accumulation and Economic Growth", en En F.A. Lutz y D.C Hague (comps.)", The Theory of Capital. Nueva York, St Martin Press.

Kaneko, A. y D. Matsuzaki (2009). "Consumption Tax and Economic Growth in an Overlapping Generations Model with Money Holdings". Journal of Economics, vol. 98, 155-175.

Kim, S. (1992). "Taxes, Growth and Welfare in an Endogenous Growth Model". Tesis doctoral no publicada. Chicago Illinois, Universidad de Chicago.

Mendoza, E. G. M. Milesi Ferreti y P. Asea (1997). “On the Ineffectiveness of Tax Policy in Altering Long Run Growth: Harberger's Superneutrality Conjecture". Journal of Public Economics, vol. 66, 99-126.

Pasinetti, L. (1960). "A Mathematical Formulation of the Ricardian System". Review of Economic Studies, vol. 28, 78-98.

Rebelo, S. (1991). "Long Run Policy Analysis and Long Run Growth". Journal of Political Economy, vol. 99, 500-521.

Sargent, T. y N. Wallace (1973). "The Stability of Models of Money and Growth". Econometrica, vol. 41, 1043-1048.

Solow, R. (1956). "A Contribution to the Theory of Economic Growth". Quarterly, Journal of Economics, vol. 70, 65-94.

Segura-Ubiergo, A. A. Simone, S. Gupta y Q. Cui (2010). “New Evidence on Fiscal Adjustment and Growth in Transition Economies". Comparative Economic Studies, vol. 52, 18-37. 
Stockey, N. y S. Rebelo (1993). “Growth Effects of Flat Rates Taxes”. NBER Working Paper 4426. National Bureau of Economic Research.

Woodford, M (2001). "The Taylor Rule and the Optimal Monetary Policy". American Economic Review, vol. 2, 232-237. 